\title{
Inadvertent laparoelytrotomy in second stage of labour: a case report
}

\author{
Shristy Mohanty*, Satyabhama Marandi, Bhismadev Chhatria
}

Department of Obstetrics and Gynaecology, IMS and SUM Hospital, Bhubaneswar, Odisha, India

Received: 17 October 2021

Revised: 09 November 2021

Accepted: 10 November 2021

\section{*Correspondence:}

Dr. Shristy Mohanty,

E-mail: shristymohanty@gmail.com

Copyright: (C) the author(s), publisher and licensee Medip Academy. This is an open-access article distributed under the terms of the Creative Commons Attribution Non-Commercial License, which permits unrestricted non-commercial use, distribution, and reproduction in any medium, provided the original work is properly cited.

\section{ABSTRACT}

Laparoelytrotomy is the accidental delivery via a vaginal incision during caesarean section in second stage of labour. It refers to the inadvertent delivery of the foetus through a transverse incision given over the vagina during a caesarean section (C-section). It is a rather uncommon complication of $\mathrm{C}$-section encountered at advanced dilation which can lead to maternal complications such as traumatic postpartum hemorrhage, injury to bladder, ureters and difficult reconstruction of vagina. Here, we present a case report of inadvertent laparoelytrotomy during C-section.

Keywords: Inadvertent laparoelytrotomy, Caesarean section, Complication

\section{INTRODUCTION}

Laparoelytrotomy is the accidental delivery via a vaginal incision during caesarean section in second stage of labour. It refers to the inadvertent delivery of the foetus through a transverse incision given over the vagina during a caesarean section (C-section). Albeit unintended, it is a rather uncommon complication of $\mathrm{C}$-section encountered at advanced dilation. ${ }^{1}$

\section{CASE REPORT}

Mrs. S., 25 year old female with an obstetric score of G2 A1 came to the labour room at 38 weeks 5 days period of gestation with the chief complaint of leaking per vagina since 2 hours.

She had a previous history of spontaneous abortion at 3 months period of gestation, 3 years back for which suction evacuation was not done.

Her present pregnancy was an intra-uterine insemination (IUI) conception and she had visited our ante-natal clinic regularly.
She was a known case of gestational diabetes mellitus on Insulin injection (diagnosed in the $7^{\text {th }}$ month of pregnancy). Her hemoglobin A1c (Hb1AC) in January 2021 was $5.7 \%$ and fasting and post-prandial blood sugar values were controlled after starting insulin aspart.

Abdominal ultrasonography done at 37 weeks 5 days period of gestation (POG) showed a $3.1 \mathrm{~kg}$ live baby in cephalic presentation with adequate liquor.

On admission, at $9.00 \mathrm{pm}$ on 07 March 2021, her uterus was term sized and relaxed. The foetal heart rate was wellappreciated as well. There was no evidence of frank leaking on vulval inspection.

Per vaginal findings on admission were: cervix $2 \mathrm{~cm}$ long, soft in consistency, mid-position; os was $1 \mathrm{~cm}$ dilated, foetal membranes were present and the presenting part (head) was at station $-2 \mathrm{~cm}$.

She was left for spontaneous progression of labour. Blood glucose level monitoring was done $8^{\text {th }}$ hourly, contractions and foetal heart rate were monitored at frequent intervals. 
7 hours after admission, i. e. at 4.00 am 08 March 2021, she was getting 2 contractions lasting for 20 seconds in 10 minutes. Per vaginal reassessment revealed cervical effacement of $50 \%$, os dilation of $2.5 \mathrm{~cm}$, membranes were present and head station was $-2 \mathrm{~cm}$.

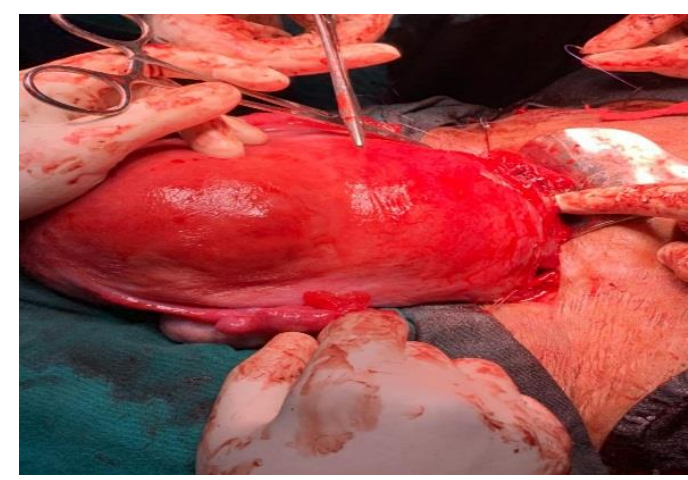

Figure 1: Bandl's ring.

At 8.00 am on 8 March 2021, she was getting 3-4 contractions lasting for seconds in 10 minutes. Vaginal reassessment showed that the cervix was fully effaced, os was $10 \mathrm{~cm}$ dilated, membranes were applied. Artificial rupture of membranes was done following which $10 \mathrm{ml}$ of meconium stained liquor drained. Small scalp folding was palpable and head station was zero.

Emergency lower segment caesarean section (LSCS) was done with the indication being "early features of obstruction with foetal distress."

\section{Intra-operative findings}

On opening the abdomen, bowel distension was visualised. The urinary bladder appeared oedematous. Uterus showed Bandl's ring formation. Transverse incision was given and baby was delivered. A $3.78 \mathrm{~kg}$ male baby was delivered by vertex at 9.17 am on 08 March 2021. Baby cried immediately after birth.

Liquor amnii was reduced and meconium staining was present. Placenta and membranes were delivered in toto.

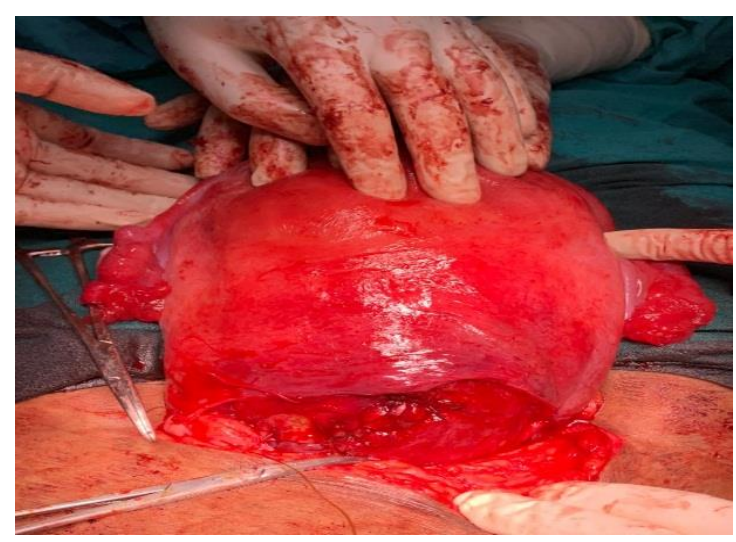

Figure 2: Incision site with extension sutured.

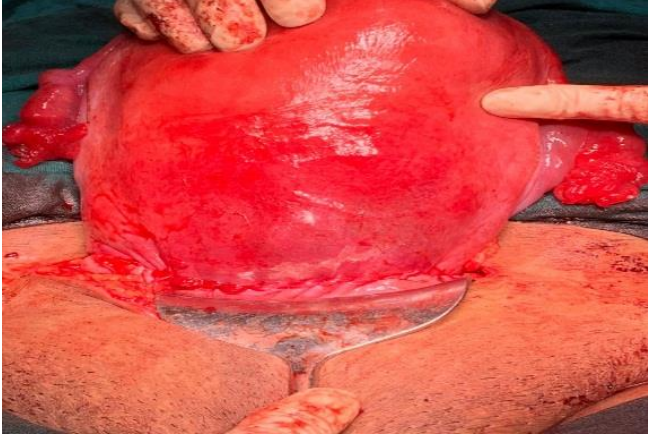

Figure 3: Uterus after suturing.

On attempting uterine closure, it was found that the incision was over the vagina.

There was complete shearing of anterior and posterior vaginal wall. Colporrhexis of anterior vaginal wall on the left side of size $5 \mathrm{~cm}$ was noted. Reconstruction was done along with bilateral uterine artery ligation. All the margins of the uterus were approximated maintaining haemostasis. Peritonisation was done. Bladder was intact. Abdominal closure was done in layers and skin was sutured by mattress sutures.

Patient was kept nil per oral for 24 hours and Foley's catheter was kept in-situ for 7 days. Post-operative period was uneventful.

\section{DISCUSSION}

This case shows how the vagina is mistaken for the lower uterine segment (LUS) while giving incision in second stage caesarean section. It is important to recognize the error in judgement and repair accordingly. But first and foremost, it is of utmost importance to avoid such errors. The anatomy of lower uterine segment should be wellunderstood to prevent such occurrences.

In advanced labor, lower uterine segment is stretched out and considerable vaginal advancement occurs. It may lead to maternal morbidity such as traumatic postpartum hemorrhage, injury to the bladder/ureters and difficulty in reconstructing the vagina. ${ }^{1-6}$

The upper uterine segment has the peritoneum adhered to it. It is quite difficult if not impossible to separate the peritoneum from the uterus here. Whereas, the lower uterine segment has a loose covering of peritoneum which is easily separable.

The transition of this adherent peritoneum to loose or "free" peritoneum is the utero-vesical fold. This landmark has to be identified because this defines where the lower uterine segment begins from.

When C-section is performed in a case of obstructed labour with full dilation of the cervix, it is quite difficult to identify the LUS. The LUS in such cases is usually 
elongated and stretched over the presenting part. Hence there is a chance of the uterine incision extending into the vagina. To avoid extensions or entry to the vagina, the upper limit of the lower uterine segment has to be properly identified. The uterine incision should be given within 2-3 $\mathrm{cm}$ below this landmark.

Possible complications are injury to the ureters directly or while securing haemostasis.

Long term follow up will be needed to see delayed postoperative complications such as dyspareunia or urinary problems. These women might need caesarean section in their next delivery also and there would probably be a lot of adhesions encountered during the future $\mathrm{C}$-sections.

\section{CONCLUSION}

In modern obstetrics transverse incision on the lower segment of the uterus is the standard procedure. So, it goes unsaid that, even in the most experienced hands, laparoelytrotomy is an accidental occurrence. Not much has been written about laparoelytrotomy in modern obstetric literature. The case described here occurred by accident due to a thinned out lower uterine segment. We think inadvertent laparoelytrotomy will continue to occur in cases of dystocia, obstructed labour with stretched lower uterine segments. The key is prompt identification of anatomical landmarks and restoration of anatomy to ensure successful outcomes.

Funding: No funding sources Conflict of interest: None declared Ethical approval: Not required

\section{REFERENCES}

1. Pereira N, Rasner JN, Brannon RK, Green MA. Inadvertent vaginal entry during cesarean delivery: A report of two cases. J Reprod Med. 2014;59:429-32.

2. Peleg D, Perlitz Y, Pansky S, Levit A, Ben-Ami M. Accidental delivery through a vaginal incision (laparoelytrotomy) during caesarean section in the second stage of labour. BJOG. 2001;108:659-60.

3. Rashid M, Rashid M. Accidental delivery of a baby during a caesarean section through a vaginal incision (a laparoelytrotomy). BMJ Case Rep. 2010; bcr0720103135.

4. Goodlin RC, Scott JC, Woods RE, Anderson JC. Laparoelytrotomy or abdominal delivery without uterine incision. Am J Obstet Gynecol. 1982;144:9901 .

5. Gortzak-Uzan L, Walsch A, Gortzak Y, Katz M, Mazor M, Hallak M, et al. Accidental vaginal incision during cesarean section. A report of four cases. J Reprod Med. 2001;46:1017-20.

6. Işik AZ, Gülmezoğlu M. Laparoelytrotomy: Abdominal delivery without uterine incision. Am J Obstet Gynecol. 1991;165:781.

Cite this article as: Mohanty S, Marandi S, Chhatria B. Inadvertent laparoelytrotomy in second stage of labour: a case report. Int J Reprod Contracept Obstet Gynecol 2021;10:4606-8. 